\title{
Rupture of the axillary (circumflex) nerve and artery in a champion jockey
}

\author{
J Patel, M Turner, R Birch, P McCrory
}

\begin{abstract}
Rupture of the circumflex artery and nerve, without fracture or dislocation, is a rare traumatic event. Such a case is reported in a champion flat jockey who sustained blunt trauma to the shoulder after a fall during a race; the literature is also reviewed. At urgent surgical exploration, the axillary artery and nerve were repaired. The patient subsequently successfully returned to professional riding. This case highlights the difficulties in diagnosis and management.

(Br F Sports Med 2001;35:361-363)
\end{abstract}

Keywords: axillary/circumflex nerve; infraclavicular brachial plexus; horse racing; jockey

\section{Case report}

A thirty five year old champion jockey fell from his horse during a two and a half mile race at Ascot Racecourse in June 2000. There was a field of 24 horses, and, on the last bend, one horse fell, bringing down three others (including the mount of this particular jockey). The jockey subsequently recalled being kicked in the left shoulder while falling (see video on http://bjsm.bmjjournals/cgi/content/full/35/5/ 361/DC1).

Within two minutes of the fall, the jockey was attended by the racecourse medical team comprising a doctor, paramedic, and paramedic ambulance. At the time, the jockey complained of severe pain in the left shoulder, and swelling was visible about the shoulder joint. A preliminary diagnosis of a fracture and/or dislocation of the left shoulder was made, and the jockey was transferred immediately to the local hospital.

On arrival, he was seen in the emergency department. On inspection, a hoof mark was noted on the posterolateral aspect of the shoulder, and a large haematoma in the axilla was seen. Sensation was absent in the proximal lateral arm and paraesthesia was present in the rest of the upper limb. Motor testing showed an absence of power in the deltoid (Medical Research Council Grade (MRC) 0/5) with normal motor function elsewhere within the limits of examination. Peripheral pulses and circulation in the upper limb were clinically normal.

Plain radiographs of the shoulder were normal. In particular, there was no radiological evidence of fracture or dislocation. A computerised tomographic scan of the shoulder (with contrast) was performed, and an undisplaced fracture of the acromion was noted, but no other abnormality was present.

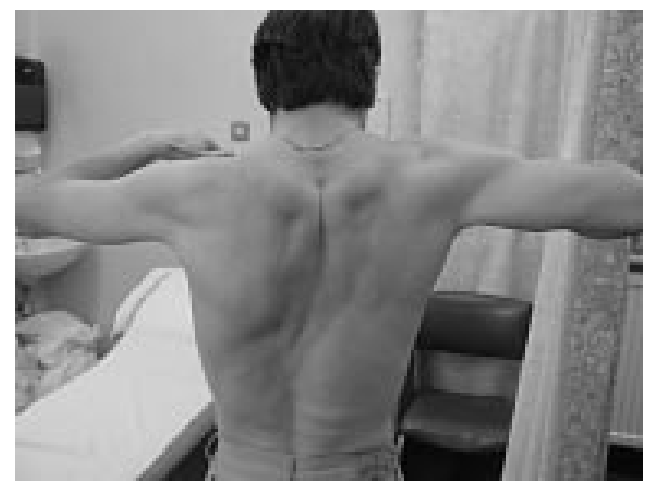

Figure 1 Posterior view of arms abducted to $90^{\circ}$. The picture was taken six months after the injury. Wasting in the left deltoid can be seen and the degree of active abduction visualised.

A lesion of the axillary nerve was suspected, and, in view of the energy of trauma and associated haematoma, an urgent exploration of the infraclavicular brachial plexus was recommended. The following day, the jockey was transferred to the Royal National Orthopaedic Hospital at Stanmore for definitive surgical management.

At operation, a haematoma of about $750 \mathrm{ml}$ was evacuated from around the shoulder. The circumflex artery had ruptured $1 \mathrm{~mm}$ from its origin from the axillary artery and was tied off. The axillary nerve was ruptured and repaired using a three stranded cable graft from the medial cutaneous nerve of the forearm. The medial and posterior cords of the brachial plexus were visibly contused. No intraoperative nerve action potentials were recorded.

Four months after the operation, a flicker of activity (MRC grade 1) was noted in the posterior fibres of the deltoid. Six months after the operation, the jockey had regained sufficient strength (deltoid anterior/middle and posterior fibres MRC grade 4-4+) in the upper limb to start professional racing. Sensation was normal (fig 1). At this time, nerve conduction studies and needle electromyography were performed. The needle electromyograph showed polyphasic potentials with a reduced interference pattern in the deltoid muscle. The nerve conduction studies showed intact compound muscle action potentials in the nerves arising from all cords of the brachial plexus. The findings were in keeping with recovery of the function of the axillary nerve.

\section{Discussion}

Injuries to the shoulder are unusual in professional horse racing. In England, for the eight year period 1992-1999 inclusive, there were 4084 flat racing meetings involving 
279792 rides and 1110 falls ( $0.4 \%$ of rides). Of the 1110 fallers, 662 were unhurt (59.6\%). During the period 1992-1999, the average number of rides undertaken by a full professional jockey was 380/year, and the highest number of rides undertaken by a flat jockey in any one year is 1317 rides. On average, a flat jockey falls every 268 rides, is injured every 673 rides, and suffers a serious injury every 3994 rides.

Of the injuries to jockeys occurring during this period, there was only one dislocation of the shoulder. Of all other significant upper limb injuries, there were eight clavicular fractures and 21 forearm fractures.

The axillary nerve arises from the posterior cord of the brachial plexus and contains fibres derived from C5 and C6 spinal nerve roots. It passes through the quadrilateral space just below the shoulder joint. The nerve then curves around the posterolateral surface of the humerus deep to the deltoid dividing into anterior and posterior branches, both of which supply the deltoid muscle. The upper lateral cutaneous nerve of the arm arises from the posterior branch and supplies the skin over the deltoid. The axillary nerve also gives a branch to supply the teres minor muscle.

Most closed axillary nerve injuries occur in association with a glenohumeral dislocation and/or fracture of the surgical neck of the humerus. ${ }^{1}$ Sometimes the axillary nerve is damaged during reduction of a shoulder dislocation. ${ }^{2}$ Blunt trauma with direct injury to the nerve has been reported in American football and ice hockey players. ${ }^{3}$ After glenohumeral dislocation, transient axillary numbness is often noted. This usually reflects a neurapraxic injury rather than nerve rupture. In this case, the coexistent axillary haematoma was the key to suspecting more serious neurovascular injury.

Recovery often occurs spontaneously with closed injuries because the lesion is typically in continuity and the nerve injury is a neurapraxia. A conservative course of management is appropriate for these injuries. ${ }^{5-7}$

Rupture of components of the infraclavicular brachial plexus without associated fracture or dislocation is a rare but recognised event following blunt trauma. ${ }^{89}$ Acute nerve ruptures should be repaired, and the outcome is far superior when the surgery is performed early. ${ }^{4}{ }^{10-13}$ Late repair of nerves is associated with increased technical difficulty principally because the nerve ends are retracted and embedded in scar tissue. In cases in which there has been an associated vascular injury, nerve stumps may be encased in a false aneurysm, further complicating the difficulty of the repair. Delayed surgery may also be unsuccessful if the denervated muscle has become fibrosed. In such situations, even with successful nerve reinnervation, the functional outcome may remain poor. ${ }^{6}$

Vascular injury associated with rupture of the axillary nerve is well recognised but uncommon. Of over 200 published cases of axillary nerve rupture, associated injury to the axillary artery (or one of its major branches) was noted in only $10-15 \%$ of cases. ${ }^{1014}$

Early diagnosis of a nerve rupture or significant nerve injury is essential for prompt surgical intervention and optimal management. Clinical features suggestive of nerve rupture are the energy of the trauma, open wound, associated vascular injury, and complete nerve lesion. The last symptom may be difficult to assess in the acute situation as most athletes with a shoulder dislocation report a dead or numb arm sensation. ${ }^{15}$ Far more useful in this situation is the documentation of impaired sensation in the cutaneous distribution of the axillary nerve over the lateral aspect of the upper arm.

It is worth noting that, in the acute situation, nerve conduction and needle electromyography may be normal even when the relevant nerve is ruptured. Electrophysiological changes take about 7-10 days to develop after nerve injury caused by nerve transection. ${ }^{76}$ In such cases, clinical suspicion of the injury is paramount.

CONCLUSION

Although shoulder injuries are uncommon in professional horseracing, suspicion of major neurovascular trauma must be considered with all such injuries given the high energy trauma involved. In this case, the clinical features immediately suggested a rupture of the axillary nerve and artery, especially when the initial plain imaging studies ruled out a shoulder fracture or joint dislocation. Early exploration and definitive primary repair are critical to the outcome of such injuries. In this case, successful functional recovery and return to professional horse racing followed urgent treatment of this injury.

1 Liveson J. Nerve lesions associated with shoulder dislocation: an electrodiagnostic study of 11 cases. $F$ Neurol Neurosurg Psychiatry 1984;47:742-4.

2 Wilbourn A, Lederman R, Sweeney P. Brachial plexopathy: a complication of reduction of shoulder dislocation. Can 7 Neurol Sci 1992;19:300-1.

3 Perlmutter G, Leffert R, Zarins B. Direct injury to the axillary nerve in athletes playing contact sports. Am $\mathcal{F}$ Sports Med 1997;25:65-8.

4 Berry H, Bril V. Axillary nerve palsy following blunt trauma to the shoulder region: a clinical and electrophysiological review. 7 Neurol Neurosurg Psychiatry 1982;45:1027-32.

5 Lefert R, Seddon H. Infraclavicular brachial plexus injuries. f Bone foint Surg [Br] 1965;47:9-22.

F Bone foint Surg $[\mathrm{Br}]$ 1965;47:9-22.
6 Seddon H. Surgical disorders of the peripheral nerves. Seddon H. Surgical disorders of the peripheral

7 Sunderland S. Nerves and nerve injuries. 2nd ed. Edinburgh: Churchill-Livingstone, 1978.

8 Hopkins G, Ward A, Ra G. Lone axillary nerve lesion due to closed non-dislocating injury of the shoulder. Injury 1985; 16:305-6.

9 MacNamara A, Ismail A. Combined brachial plexus and vascular injury in the absence of bony injury. $f$ Accid Emerg Med 2000;17:3780-9.

10 Bonnard C, Anastakis D, van Melle G, et al. Isolated and combined lesions of the axillary nerve: a review of 146 cases. F Bone Foint Surg [Br] 1999;81:212-17.

11 Cavanagh J, Bonney G, Birch R. The infraclavicular brachial plexus: the case for primary repair. F Bone foint Surg $[\mathrm{Br}]$ 1987;69:489.

12 Alnot J, Liverneaux P, Silberman O. Lesions to the axillary nerve. Revue de Chirurgie Orthopaedique 1996;82:579-89.

13 Petrucci F, Morelli A. Axillary nerve injuries: 21 cases treated by nerve graft and neurolysis. F Hand Surg 1982;7:271-8.

14 Birch R, Bonney G, Wynn Parry C. Axillary nerve trauma. In: Seddon H, ed. Surgical disorders of the peripheral nerves. London: Churchill-Livingstone, 1998:146-7.

15 Brukner P, Khan KM. Clinical sports medicine. 2nd ed. Sydney: McGraw-Hill, 2000

16 Stewart J. Focal peripheral neuropathies. 3rd ed. Philadelphia: Lippincott Williams \& Wilkins, 2000 


\section{Take home message}

Nerve injuries caused by high energy trauma are likely to be ruptures, particularly if associated with an arterial injury. Optimal outcome of these injuries can be achieved with prompt clinical diagnosis and urgent repair.

\section{Commentary}

I congratulate the authors on a functionally good outcome in this case, but a few points on traumatic plexus injury require further comment.

Injuries to the brachial plexus are best categorised as "in continuity" or "not in continuity" (or transections). A nerve can be transected sharply, as in a sharp knife injury, or, more commonly, there is blunt transection (such as a kick from a horse). Blunt transection results in nerve injury not only at the site of transection, but injury extends proximally and distally along the nerve for a variable distance, depending on the degree of associated "stretch" injury and contusion. Macroscopic and electrophysiological delineation of this extra length of injury takes a few weeks to occur. Therefore repair of a blunt transection should not be performed earlier than two weeks after such an injury. When immediate repair of associated injuries occurs, such as repair of the arterial injury described here, and a transected nerve is identified, the nerve ends should be sutured to the fascial layer to prevent nerve retraction, and repair delayed for two weeks. At the time of subsequent repair, the injury will be macroscopically apparent, and the nerve is cut back to healthy looking fascicles before suture or grafting. Although the graft appears to have taken in this case, the authors make no mention of the nerve gap, length of nerve cut back, or the graft length. As a general rule, results will not always be as successful as this if allowance is not made for the stretch injury proximal and distal to a blunt transection.

The association with arterial injury is not uncommon in the setting of brachial plexus injuries, and consideration should always be given to performing angiography before the operation in all cases with haematoma formation, laceration, or gunshot wound. If needed, the services of a vascular surgeon can then be organised in advance. In the case presented, an angiogram should have been performed before surgery given the immediate onset haematoma.

Finally, I must disagree with the authors' assertion in the discussion that a "complete nerve lesion" is suggestive of nerve rupture. To the contrary, most complete nerve injuries seen in the acute setting are neurapraxic (Sunderland grade I) and ultimately recover with conservative treatment. In the same paragraph, the authors state that cutaneous sensory loss in axillary nerve territory in the acute setting is also suggestive of nerve rupture. Again, I must stress that, in most cases, this is due to neurapraxia and not rupture (Sunderland grade V). Only careful clinical and electrophysiological follow up can differentiate a Sunderland I from higher grades of injury.

G A DAVIS

Consultant Neurosurgeon, Department of Neurosurgery, Austin and Repatriation Medical Centre, Burgundy Street, Heidelberg, Victoria 3084, Australia gadavis@netspace.net.au 